\title{
A new approach for the treatment of CLL using chlorambucil/hydroxychloroquine-loaded anti-CD20 nanoparticles
}

\author{
Sara Capolla ${ }^{1, \S}(\bowtie)$, Nelly Mezzaroba ${ }^{1, \S}$, Sonia Zorzet ${ }^{1}$, Claudio Tripodo ${ }^{2}$, Ramiro Mendoza-Maldonado ${ }^{3}$, \\ Marilena Granzotto ${ }^{4}$, Francesca Vita ${ }^{1}$, Ruben Spretz ${ }^{5}$, Gustavo Larsen ${ }^{5,6}$, Sandra Noriega ${ }^{5}$, Eduardo Mansilla ${ }^{7}$, \\ Michele Dal Bo ${ }^{8}$, Valter Gattei ${ }^{8}$, Gabriele Pozzato ${ }^{4}$, Luis Núñez ${ }^{5,6}$, and Paolo Macor ${ }^{1,9}(\varangle)$ \\ ${ }^{1}$ Department of Life Sciences, University of Trieste, 34127 Trieste, Italy \\ ${ }^{2}$ Department of Human Pathology, University of Palermo, 90133 Sicilia, Italy \\ ${ }^{3}$ Molecular Oncology Unit, National Laboratory Consorzio Interuniversitatio per le Biotecnologie (CIB), 34012 Trieste, Italy \\ ${ }^{4}$ Dipartimento Universitario Clinico di Scienze mediche, Chirurgiche e della Salute, University of Trieste, 34149 Trieste, Italy \\ ${ }^{5}$ LNK Chemsolutions LLC, Lincoln, NE 68521, USA \\ ${ }^{6}$ Bio-Target Inc., Chicago, IL 60637, USA \\ ${ }^{7}$ Centro Ünico Coordinador de Ablacion e Implante Provincia de Buenos Aires (C.U.C.A.I.B.A.), Ministry of Health, La Plata, Buenos Aires, \\ Argentina \\ ${ }^{8}$ Clinical and Experimental Onco-Hematology Unit, Centro di Riferimento Oncologico, Istituto di Ricerca e Cura a Carattere Scientifico \\ (I.R.C.C.S.), 33081 Aviano, Italy \\ ${ }^{9}$ Callerio Foundation Onlus, Institutes of Biological Researches, 34127 Trieste, Italy \\ $\S$ These authors contributed equally to this work.
}

Received: 16 July 2015

Revised: 27 October 2015

Accepted: 30 October 2015

(C) Tsinghua University Press and Springer-Verlag Berlin Heidelberg 2015

\section{KEYWORDS}

chronic lymphocytic

leukemia,

immune targeted

nanoparticles,

treatment, xenograft model

\begin{abstract}
Current approaches for the treatment of chronic lymphocytic leukemia (CLL) have greatly improved the prognosis for survival, but some patients remain refractive to these therapeutic regimens. Hence, in addition to reducing the long-term sideeffects of therapeutics for all leukemia patients, there is an urgent need for novel therapeutic strategies for difficult-to-treat leukemia cases. Due to the cytotoxicity of drugs, the major challenge currently is to deliver the therapeutic agents to neoplastic cells while preserving the viability of non-malignant cells. In this study, we propose a therapeutic approach in which high doses of hydroxychloroquine and chlorambucil were loaded into biodegradable polymeric nanoparticles coated with an anti-CD20 antibody.We first demonstrated the ability of the nanoparticles to target and internalize in tumor B-cells. Moreover, these nanoparticles could kill not only p53-mutated/deleted leukemia cells expressing a low amount of CD20, but also circulating primary cells isolated from chronic lymphocytic leukemia patients. The safety of these nanoparticles was also demonstrated in healthy mice, and their therapeutic effects were shown in a new model of aggressive leukemia. These results showed that anti-CD20 nanoparticles containing hydroxychloroquine and chlorambucil can be effective in controlling aggressive leukemia and provided a rationale for adopting this approach for the treatment of other B-cell disorders.
\end{abstract}

Address correspondence to Sara Capolla, caposara@gmail.com; Paolo Macor, pmacor@units.it 


\section{Introduction}

Chronic lymphocytic leukemia (CLL) is a heterogeneous disease with highly variable clinical courses and survivals ranging from months to decades. In particular, a subset of patients is affected by a highrisk CLL form that rapidly progresses and develops into a disease that requires symptomatic treatment [1]. Over-represented in this group are patients bearing mutations/deletion of the TP53 gene [2]. Moreover, a high-risk CLL patient fraction was confirmed to carry mutations/deletion of other genes, such as NOTCH1, BIRC3, or SF3B1 [3-7].

For years, the standard therapy was based on the use of alkylating agents, with negligible (if any) effects on the natural history of CLL. The introduction of fludarabine signified an important breakthrough in CLL therapy. The use of monoclonal antibodies (antiCD20, anti-CD52, etc.) opened a new perspective for overcoming the paradigm of treating only patients with minimal complications; alone or in combination with chemotherapy, these therapeutics significantly increased the overall survival of the patients $[8,9]$. More recently, inhibitors of B-cell receptor signaling showed efficacy in a subset of CLL patients [9-11]. Despite the advancements in combined therapy, CLL remains an incurable disease in most cases since complete molecular remission is unachievable and, as a consequence, the disease relapses invariably after some months or years. In particular, the small subgroup of patients, known as the ultra-high-risk CLL group, shows poor response to chemo-immunotherapy and a life expectancy of less than 2 to 3 years with conventional regimens [10, 12]. These considerations indicate that new therapeutic approaches are needed to obtain complete recovery or at least to improve the survival of CLL patients. Since most patients are old and often have several co-morbidities, any new treatment approaches, in addition to higher efficacy, must be non-toxic to organs and tissues. The development of nanoparticles made with biodegradable biopolymers and loaded with chemotherapeutic agents is an attractive method to target neoplastic cells [13-15]. In fact, nanoparticles can be designed by attaching specific antibodies on their surface, so that they are able to recognize tumor-associated antigens and induce specific homing on the neoplastic cell surface [16, 17]. Therefore, the efficacy of high-dose chemotherapy is associated with the specificity and low side-effects of antibody-based therapy and the protective nature of polymeric encapsulated chemotherapeutics.

On these principles, we developed biodegradable nanoparticles (BNPs) coated with an anti-CD20 antibody to target neoplastic B-cells, and loaded them with hydroxychloroquine (HCQ) and chlorambucil (CLB) to specifically kill tumor B-cells $[18,19]$. To the best of our knowledge, this is the first report of the safety and therapeutic effects of targeted nanoparticles in a new leukemia xenograft SCID mice model.

\section{Experimental}

\subsection{Cells, antibodies, and sera}

The CLL-like cell line MEC1 [20] (kindly provided by Prof. Josee Golay), carrying both a TP53 mutation (i.e. c.422insC) and the $17 \mathrm{p} 13$ deletion, was cultured in RPMI-1640 medium (Sigma-Aldrich, Milan, Italy) supplemented with $10 \%$ fetal bovine serum (FBS; GE Healthcare Milan, Italy). Heparinized peripheral blood samples were obtained from untreated CLL patients at the University Hospital in Trieste (B-cells more than $90 \%$ of total circulating cells) after obtaining written informed consent. The study was approved by the IRB of the CRO (IRCCS) of Aviano (IRB-062010). The mononuclear cell fractions were isolated by centrifugation on Ficoll-Hypaque (GE Healthcare) density gradients [21]. MEC1 cells were suspended in serum-free RPMI-1640 medium and stained with Vybrant ${ }^{\mathrm{TM}} \mathrm{DiO}$ cell-labeling solution (GE Healthcare) as described previously in Ref. [22]. The anti-CD20 chimeric antibody Rituximab (Roche, Milan, Italy) was derived from the clinic (University of Trieste, Italy). The mouse mAb to CD20 and anti-PARP1 antibody were purchased from BioLegend (San Diego, CA) and Bethyl Laboratories, respectively. For immunophenotypical characterization, anti-human CD5 PE (Immunotools, Friesoythe, Germany), anti-human CD20 (clone L26, Novacastra), anti-human CD45 APC (Invitrogen, Milan, Italy), and anti-human CD19 TC (GE Healthcare) mAbs were used. Anti-LC3, anti- $\alpha-$ tubulin mAbs, and all the secondary antibodies were 
purchased from Sigma-Aldrich (Milan, Italy) or Aczon (Monte San Pietro, Bologna, Italy). Human sera from $\mathrm{AB} \mathrm{Rh}+$ blood donors (NHS-normal human serum) were kindly provided by the Blood Transfusion Center (Trieste, Italy) as a source of complement (NHS).

\subsection{BNP preparation}

BNPs were prepared using chemicals of reagent grade or above. Polyethylene glycol (PEG) was purchased from Nektar, San Carlos, CA; HCQ sulfate and CLB were purchased from ACROS (Belgium) and Sigma Aldrich (St Louis, MO), respectively. BNPs based on carboxylic acid terminated biodegradable polymers (PLA-b-PEG-COOH and PCL-COOH) were prepared with an average diameter of $250 \mathrm{~nm}$, as measured by dynamic light scattering (data not shown) in an under class 100 clean room conditions by implementing Bio-Target's technology at LNK Chemsolutions, LLC laboratories [19, 23]. All the BNPs (final concentration of $900 \mu \mathrm{g} / \mathrm{mL}$ ) were resuspended in PBS buffer ( $\mathrm{pH}=$ 7.4) with $10 \%$ BSA. The BNPs were diluted in serumfree RPMI-1640 medium and stained with fluorescein isothiocyanate isomer I (Sigma-Aldrich) or FluoroLinkTM Cy5.5 Monofunctional Dye (GE Healthcare).

\subsection{Animals}

Female SCID mice (4-6 weeks of age) were provided by Charles River (Milan, Italy) and maintained under pathogen-free conditions. The animals were pretreated with cyclophosphamide $(200 \mathrm{mg} / \mathrm{kg})$, inoculated subcutaneously with $10^{7}$ MEC1 cells or intravenously with $5 \times 10^{5}$ MEC1 cells after $24 \mathrm{~h}$, and examined twice weekly up to 125 days. C57/BL mice were obtained from the animal house of the University of Trieste. All the experimental procedures involving animals were performed in compliance with the guidelines of the European and the Italian laws and were approved by the Italian Ministry of Health as well as by the Administration of the University Animal House (Prot. 42/2012).

\subsection{Cytometric analysis}

The binding of the BNPs was assayed by incubating $10 \mu \mathrm{L}$ of BNPs with $5 \times 10^{5} \mathrm{MEC} 1$ cells for $1 \mathrm{~h}$ at $37^{\circ} \mathrm{C}$.
MEC1 localization in mouse blood was performed using anti-CD45 APC and anti-CD19 TC antibodies at 28 days after cell injection. For these measurements, 30,000 events were acquired using FACSCalibur (Becton Dickinson, San Jose, CA) flow cytometer and data were analyzed using CELLQuest software (Becton Dickinson) [24].

\subsection{Transmission electron microscopic analysis}

Samples were fixed for $1 \mathrm{~h}$ in a solution of $2 \%$ glutaraldehyde (Serva, Heidelberg, Germany) in $0.1 \mathrm{M}$ cacodylate buffer $(\mathrm{pH}=7.3)$ containing $0.03 \mathrm{M} \mathrm{CaCl}_{2}$, rinsed three times (10 min each wash) and postfixed in $1 \%$ osmium tetroxide for $1 \mathrm{~h}$ at $4{ }^{\circ} \mathrm{C}$. Samples were then dehydrated in ethanol of increasing gradients to $100 \%$ and embedded in Dow Epoxy Resin (DER 332; Unione Chimica Europea, Milan, Italy) and DER732 (Serva), as previously described by Zabucchi et al. [25]. Ultrathin sections were cut using an ultratome Leica Ultracut UCT8 (Leica, Wirn, Austria), double stained with uranyl acetate and lead citrate and observed in a transmission electron microscope (EM 208, Philips, Eindhoven, The Netherlands). Micrographs were taken with a Morada Camera (Olympus, Munster, Germany).

\subsection{Cell viability, apoptosis, and autophagy}

To investigate the ability of BNPs to affect cell viability, MEC1 cells $\left(2 \times 10^{5}\right)$ were incubated with different concentrations of BNPs for $48 \mathrm{~h}$ at $37{ }^{\circ} \mathrm{C}$ (in a humidified $37^{\circ} \mathrm{C}, 5 \% \mathrm{CO}_{2}$ incubator). The amount of residual viable cells was determined using the MTT assay [26] and the percentage of dead cells was calculated as 100× [(test release - spontaneous release)/ (total release - spontaneous release)]. Apoptosis of the patient's B cells was measured using FITC-labeled recombinant human Annexin $\mathrm{V}$ assay (Apoptosis detection kit, Immunostep, Spain) according to the manufacturer's instructions. For each measurement, 30,000 events were acquired using a standard FACSCalibur (Becton Dickinson) flow cytometer and data were analyzed using CellQuest (Becton Dickinson). PARP-1 and LC-3 activation were evaluated by immunoblotting to study apoptosis induction and autophagy impairment, respectively [27]. 


\subsection{Complement-mediated lysis}

A previously described procedure of complementdependent cytotoxicity (CDC) measurements with some modifications was used to evaluate the effect of Rituximab® ${ }^{\circledR}$ on complement-mediated killing of tumor B-cells [28]. The number of residual viable cells was estimated using the MTT assay.

\subsection{Blood analysis}

Red and white blood cells and platelets from treated and untreated mice were analyzed using ABX Micros E660 OT/CT (Horiba ABX Diagnostic, Montpellier, France). Other parameters in the animal plasma were analyzed using Integrated System Dx 880 (Beckman Coulter).

\subsection{Histopathological and immunohistochemical analysis}

Liver, spleen, kidney, brain, spinal cord, and bone marrow samples from leukemia-bearing mice either dead from the tumor or sacrificed at day +120 from cells injection were obtained at necropsy. For morphologic evaluation, the specimens were fixed in $10 \%$ bufferedformalin solution and embedded in paraffin. Four micrometer-thick sections were stained with H\&E. Four- to six-micrometer thick sections were fixed in cold 100\% methanol for $15 \mathrm{~min}$. Immunohistochemical analysis was performed using the avidin-biotinperoxidase complex method according to standard procedures [29], and the slides were examined under a Leica DM2000 optical microscope.

\subsection{Statistical analysis}

The data were expressed as mean \pm SD and analyzed for statistical significance by the two-tailed Student's $\mathrm{t}$ test to compare two paired groups of data. The KaplanMeier product-limit method was used to estimate survival curves and the log-rank test was adopted to compare different groups of mice.

\section{Results and discussion}

\subsection{Anti-CD20 BNPs target tumor B-cells}

Current treatment strategies for leukemia involve chemotherapy, immunotherapy, bone marrow transplant, and several new target therapies. These treatments often induce long-term side-effects, resulting in impairment of vital physiological functions among the survivors. This is particularly true for elderly/unhealthy CLL patients. While current treatment approaches have greatly improved the prognosis for survival, some patients remain refractive to current therapeutic regimens. Hence, in addition to reducing the longterm residual side-effects of therapeutics for all leukemia patients, there is an urgent need for novel therapeutic strategies for difficult-to-treat leukemia cases. Due to the cytotoxicity of drugs, the major challenge currently is to deliver the therapeutic agent to neoplastic cells while preserving the viability of non-malignant cells. Research on the use of nanoparticles as drug carriers has advanced to focus on assessing the safety and efficacy of such drug delivery systems. In this study, four different types of polymeric nanoparticles, BNP0, BNP1, BNP2, and BNP3, were prepared as in Fig. S1 (in the Electronic Supplementary Material (ESM)) and were characterized as previously described in Ref. [19]. The BNP0 was prepared only using polymeric carriers (PLA-b-PEG-COOH and PCL-COOH); BNP1 was prepared by conjugating the anti-CD20 chimeric antibody on the surface of BNP0; BNP2 was prepared by encapsulating the HCQ sulfate and CLB inside the core of BNP1m while BNP3 was prepared by loading chemotherapeutic drugs inside BNP0. To characterize both untargeted and targeted nanoparticles, transmission electron microscopy (TEM) and dynamic light scattering were used. In details, TEM showed that untargeted nanoparticles have a core diameter of $110 \pm 40 \mathrm{~nm}$, while anti-CD20-conjugated nanoparticles have a core diameter of $90 \pm 30 \mathrm{~nm}$. For dynamic light scattering analysis, untargeted nanoparticles have an hydrodynamic diameter of $190 \pm 60 \mathrm{~nm}$, while targeted nanoparticles have a diameter of $230 \pm 70 \mathrm{~nm}$; moreover, the $\zeta$-potential evidenced values of $-7.8 \pm 0.9$ and $-6.0 \pm$ $0.6 \mathrm{mV}$ for untargeted and anti-CD20 conjugated BNPs respectively, as previously described in Ref. [19]. During the experiments, nanoparticles were stored at -20 and $+4{ }^{\circ} \mathrm{C}$ and then tested. We did not observe any significant modification in their morphology and their capacity to target and kill tumor B-cells, both in vitro and in vivo, which suggested that they could 
remain stable for almost 1 year after their production. To characterize the effect of the BNPs, the CLL-like MEC1 cell line was used. It was initially purified from a CLL patient [20] and carried a mutation in the TP53 gene and the $17 \mathrm{p} 13$ deletion, as demonstrated by direct sequencing and FISH analysis (data not shown). Moreover, its immunophenotype was studied by cytometric analysis, thus confirming what previously reported in literature [30]. In fact, more than $95 \%$ of the MEC1 cells highly expressed human markers like CD20 and CD45, but CD5 expression was not detected (Fig. S2 in the ESM). The functional characterization of the BNPs was started by evaluating their ability to bind to leukemia cells. To this end, BNP0 and BNP1 were labeled with FITC and added to the MEC1 cells at different incubation times. BNP1 could target MEC1 cells in a dose- and time-dependent manner with a maximal uptake after $1 \mathrm{~h}$ of incubation and using $10 \mu \mathrm{L}$ of the particles. Under these conditions, $74 \%$ of the cells appeared tagged by BNP1 (Fig. 1(a)). On the contrary, BNP0 did not demonstrate specific binding after $1 \mathrm{~h}$ incubation, suggesting the importance of the anti-CD20 antibody in the targeting of the B-cells by the BNPs.

The interaction of the BNPs with MEC1 cells was further confirmed by confocal microscopy images by incubating cells with BNPs labeled with FAST-DiO and Cy5.5, respectively (Fig. S3 in the ESM). Moreover, TEM studies were performed to follow the migration of the BNPs into tumor B-cells. Two different types of BNPs were prepared as shown in Fig. S1 (in the ESM), and these were labeled as BNPA and BNPB. BNPA was prepared by encapsulating gadopentetate dimeglumine (Magnevist H, Bayer HealthCare Pharmaceuticals Inc) while BNPB was prepared by conjugating the antiCD20 antibody to the surface of BNPA. By exploiting the presence of $\mathrm{Gd}$ in the particles, the migration of the BNPs was followed by TEM analysis by incubating the MEC1 cells with these two different types of BNPs (Fig. S1 in the ESM).

In detail, BNPA was never seen inside the cells (data not shown) while images showed the binding of BNPB and its interaction with the cell surface. Moreover, BNPs were never documented in the nucleus and the absence of vesicles surrounding them suggested the internalization of the BNPs through a process different (a)
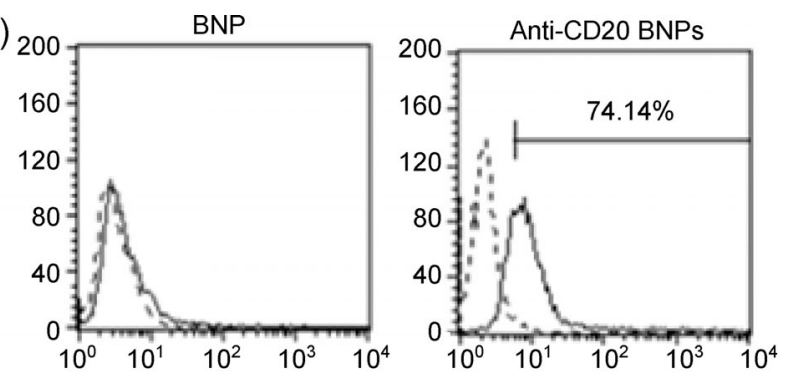

(b)

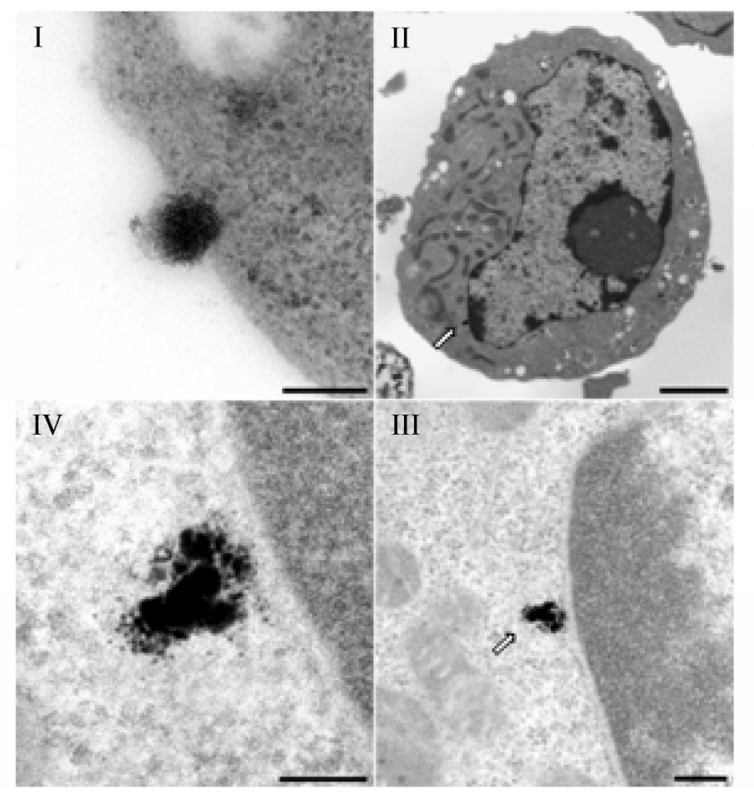

Figure 1 Interaction between tumor B-cells and BNPs. (a) Binding of anti-CD20 BNPs to MEC1 cells. MEC1 cells were incubated with FITC-labeled BNPs for $1 \mathrm{~h}$ at $37^{\circ} \mathrm{C}$ and analyzed using FACS. FL1-H, green fluorescence, $530 / 30 \mathrm{~nm}$ bandpass filter. (b) Internalization of anti-CD20 BNPs to MEC1 cells. MEC1 cells were incubated with BNPB for $1 \mathrm{~h}$ and analyzed by TEM: Ultrastructural appearance of MEC1/BNP1 interaction and internalization was documented. Arrows in bII and bIII indicate typical cytoplasmic localization of anti-CD20 BNP. Bars represent $200 \mathrm{~nm}$ ((b)I, (b)III), $2 \mu \mathrm{m}$ ((b)II), and $100 \mathrm{~nm}$ ((b)IV).

from endocytosis (Fig. 1(b)). This data confirmed the results previously obtained both in vitro and in vivo and demonstrated the importance of a targeting agent as an anti-CD20 recombinant antibody on the nanoparticle surface [18]. The internalization of BNPs outside endosomes has already been demonstrated for other tumor B-cell lines incubated with BNPs [18], which passed through the membrane without significantly disrupting it.

\subsection{BNP2 induces tumor B-cell cytotoxicity}

In this study, CLB and HCQ were loaded inside 
polymeric nanoparticles because of their synergistic effect against cancer B-cells, as we have previously described [18]. In particular, CLB is an alkylating agent administered orally, whose rate of absorption can vary significantly from patient to patient, thus causing side-effects in some patients [31, 32]. In addition, most B-cell malignancies will become resistant to CLB at some point, irrespective of whether it is used at increasing doses or within more aggressive regimens. In resistant situations, it could be important to have a therapeutic system for better delivery of high amounts of drugs specifically inside malignant B-cells in order to circumvent genetically driven mechanisms of tumor resistance. The combination of an elevated concentration of CLB intracellularly with another kind of cytotoxic drug not dependent on surviving genes could not only enhance their respective toxic activities, but perhaps make a resistant leukemia cell sensitive again. HCQ has demonstrated an interesting cytotoxic effect depending on its capacity to block autophagosomes/ lysosome fusion. Its anti-neoplastic properties in vitro depend on its concentration; however, this property is unobtainable in vivo by the usual oral administration route [33-36]. The synergistic effect of HCQ and CLB was previously described by our group [18] and it could be important, especially for CLL patients in an already resistant disease state, or in those with poor prognostic biological characteristics. These drugs together could cause high cytotoxic effects by mainly inducing autophagy and apoptosis [18]. In order to evaluate the cytotoxic effect of BNPs, MEC1 cells were incubated with different amounts of BNP0, BNP1, BNP2, and BNP3 for $48 \mathrm{~h}$ and the residual viable cells were measured. Particles loaded with chemotherapeutic drugs, such as BNP2 and BNP3, could induce cytotoxicity in a dose-dependent manner while empty particles, such as BNP1, were almost ineffective. Furthermore, $2 \mu \mathrm{L}$ of BNP2 or BNP3 was sufficient to kill more than $85 \%$ of the MEC1 cells, suggesting that the chemotherapeutic drugs maintained their cytotoxic properties even after encapsulation inside the nanoparticles. On the contrary, treatment with BNP1 killed less than $20 \%$ of the cells in this in vitro test, showing that this approach had good safety (Fig. 2(a)). The cytotoxicity is due to the pro-apoptotic effect of the chemotherapeutic drugs. Forty-eight hours of
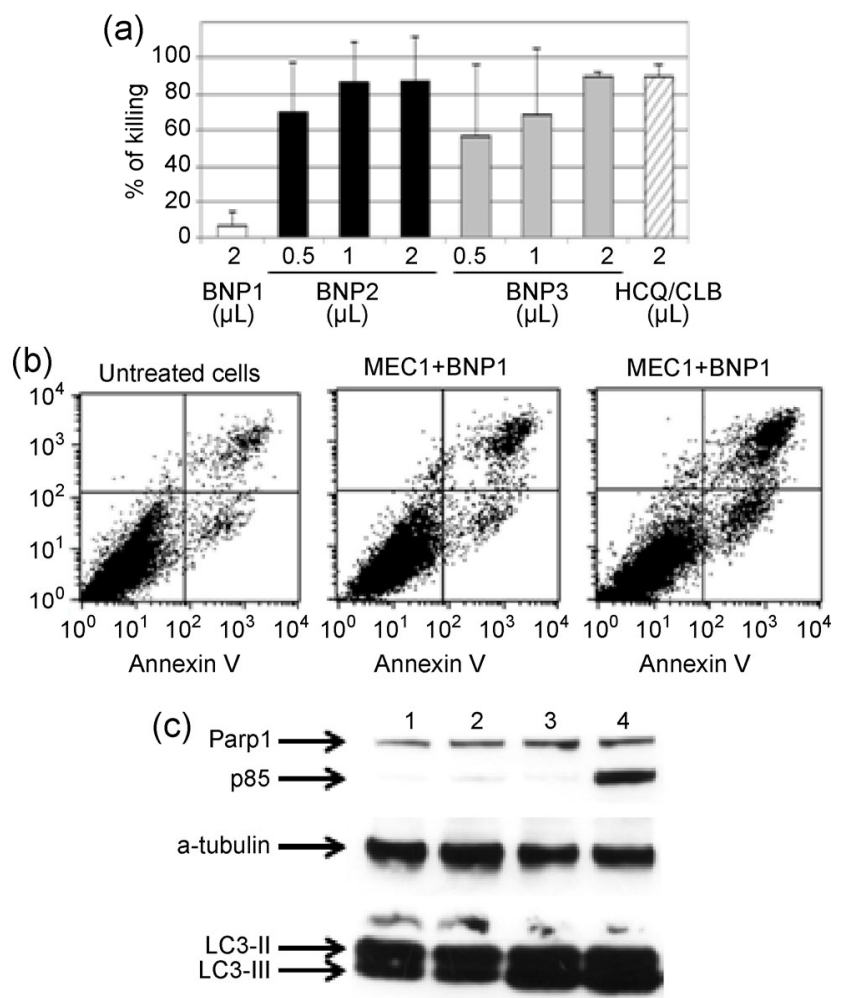

Figure 2 In vitro characterization of the cytotoxic effect of BNP2. MEC1 cells (a) were incubated with $0.5,1$, and $2 \mu \mathrm{L}$ of BNPs or $\mathrm{HCQ}+\mathrm{CLB}$ for $48 \mathrm{~h}$ at $37{ }^{\circ} \mathrm{C}$ and residual viable cells were measured. Data are expressed as mean \pm SD. (b) MEC1 cells were incubated with $1 \mu \mathrm{L}$ of BNPs for only $16 \mathrm{~h}$ at $37^{\circ} \mathrm{C}$ and apoptotic cells were analyzed using AnnexinV/7AAD test. (c) Western blot analysis of activated PARP-1 and LC3 accumulation from cell lysates obtained from MEC1 cells incubated with 1: saline; $2: 2 \mu \mathrm{L}$ of BNP1; 3: $0.5 \mu \mathrm{L}$ of $\mathrm{BNP} 2 ; 4: 2 \mu \mathrm{L}$ of BNP2.

incubation of the cells and particles loaded with chemotherapeutic drugs resulted in a high percentage of cell destruction, avoiding any possible molecular studies. Thus, only $16-\mathrm{h}$ incubations were performed to further study apoptosis induction and autophagy impairment. In this setting, more than $20 \%$ of the tumor cells $\left(2 \times 10^{5}\right)$ incubated with $2 \mu \mathrm{L}$ of BNP2 showed an apoptotic profile in an AnnexinV/7AAD test (Fig. 2(b)). To confirm apoptosis, the poly-(ADP-ribose) polymerase (PARP-1) was visualized. The enzyme is cleaved from a 113-kDa molecule to fragments of 89 and $24 \mathrm{kDa}$ during apoptosis. PARP-1 cleavage was detected by western blot assay using cell lysates of the MEC1 cells incubated with different amounts of BNP2 for $16 \mathrm{~h}$. These apoptotic studies revealed that BNP2 could induce PARP-1 cleavage, in particular using $2 \mu \mathrm{L}$ of particles with $5 \times 10^{5}$ cells (Fig. $2(\mathrm{c})$ ). 
The same pattern was found in the study of autophagy, which was impaired by HCQ. This mechanism was analyzed by taking into consideration the LC3 protein, which is processed to a cytosolic version (LC3-I, 18-kDa) and then converted to active forms commonly used as a marker of autophagosome accumulation. The effect of HCQ on MEC1 cells was detected by evaluating the increase in the amount of LC3-III in lysates of cells incubated for only $16 \mathrm{~h}$ with 0.5 or $2 \mu \mathrm{L}$ of BNP2 by western blot analysis, also in the presence of significant basal level in untreated cells (Fig. 2(c)).

\subsection{Comparison between cytotoxic effects of BNP2 and rituximab}

Rituximab can mainly activate the complement system and also induces antibody-dependent cytotoxicity (ADCC) but has a very low killing effect due to its ability to activate apoptotic pathways. For this reason, we compared the killing of MEC1 cells induced by a saturating concentration of rituximab through complement-dependent killing, or by BNP2 acting through apoptosis/authophagy. MEC1 cells were analyzed using anti-CD20 mAb, showing high amounts of the antigen on the cell surface (mean fluorescence intensity-MFI: 784). In this particular setting, rituximab killed up to $22 \%$ of the MEC1 cells while BNP2 killed $87 \%$ of these leukemia cells (Table 1 ). The cytotoxic effect of BNP2 was evidenced by analyzing purified cells from CLL patients. Circulating CLL B-cells expressed a lower amount of CD20 on their surface compared to the MEC1 cells (MFI: 50.6 vs. 784), as observed in cells purified from 31 different untreated CLL patients already characterized for the main biological prognosticators (Table S1 in the ESM). Moreover, as shown in Table 1, the cytotoxic effect of rituximab on purified CLL cells ranged between 0 and $38 \%$, with a median value of $4.2 \%$. On the contrary, BNP2 killed up to $84 \%$ CLL cells, with a median value of $55.1 \%$ (BNP2 vs. rituximab: $p<0.00001$ ), and only 1 out of 31 patients did not respond to the treatment. Interestingly, it was also possible to perform these studies in whole blood samples, as a predictive functional test. Apoptosis was evaluated using the Annex V/7AAD test after $16 \mathrm{~h}$ of incubation with BNP2. In all the samples, more than $98 \%$ of the CD5/CD19-
Table 1 Comparison between the effects of BNP2 and rituximab

\begin{tabular}{|c|c|c|c|}
\hline & $\begin{array}{l}\text { CD20 } \\
\text { (MFI) }\end{array}$ & $\begin{array}{c}\text { BNP2 } \\
\text { (\% of killing) }\end{array}$ & $\begin{array}{l}\text { RITUXIMAB } \\
\text { (\% of killing) }\end{array}$ \\
\hline MEC1 & 784 & 86.4 & 22.0 \\
\hline Pt 1 & 73.6 & 77.4 & 0.0 \\
\hline Pt 2 & 20.2 & 26.9 & 1.4 \\
\hline Pt 3 & 22.2 & 81.7 & 8.9 \\
\hline Pt 4 & 76.2 & 25.4 & 38.0 \\
\hline Pt 5 & 65.9 & 59.6 & 9.1 \\
\hline Pt 6 & 24.1 & 0.0 & 0.0 \\
\hline Pt 7 & 38.4 & 68.4 & 0.0 \\
\hline Pt 8 & 54.8 & 65.4 & 1.9 \\
\hline Pt 9 & 33.5 & 54.1 & 0.0 \\
\hline Pt 10 & 38.9 & 39.4 & 10.6 \\
\hline Pt 11 & 32.7 & 71.7 & 3.5 \\
\hline Pt 12 & 58.0 & 58.8 & 6.2 \\
\hline Pt 13 & 35.4 & 78.8 & 0.0 \\
\hline Pt 14 & 28.0 & 84.4 & 0.0 \\
\hline Pt 15 & 33.0 & 64.0 & 0.0 \\
\hline Pt 16 & 25.3 & 55.1 & 9.9 \\
\hline Pt 17 & 42.4 & 88.9 & 31.1 \\
\hline Pt 18 & 41.4 & 44.9 & 18.0 \\
\hline Pt 19 & 64.5 & 47.9 & 21.8 \\
\hline Pt 20 & 60.5 & 61.3 & 2.9 \\
\hline Pt 21 & 50.6 & 10.3 & 2.7 \\
\hline Pt 22 & 75.5 & 32.6 & 0.0 \\
\hline Pt 23 & 44.5 & 23.4 & 0.0 \\
\hline Pt 24 & 60.0 & 29.6 & 12.6 \\
\hline Pt 25 & 53.6 & 12.9 & 9.9 \\
\hline Pt 26 & 289.1 & 72.3 & 9.3 \\
\hline Pt 27 & 64.1 & 28.4 & 0.0 \\
\hline Pt 28 & 40.2 & 41.0 & 25.6 \\
\hline Pt 29 & 63.9 & 76.1 & 5.1 \\
\hline Pt 30 & 77.7 & 63.3 & 4.2 \\
\hline Pt 31 & 82.2 & 18.2 & 10.4 \\
\hline Median (Pt) & 50.64 & 55.1 & 4.2 \\
\hline
\end{tabular}

CLL patients (Pt); MFI: mean fluorescence intensity: mean; percentage of killing: mean $(n=3)$.

positive BNP2-treated cells showed an apoptotic state, as against only $11 \%$ of the BNP1-treated cells showing apoptosis. These results seem to be independent of 
CD20 expression or of specific biological features; in fact, TP53-mutated/deleted or NOTCH1-mutated cells of patients who usually have poor response to standard therapies (Table S2 in the ESM) were anyway killed by BNP2.

\subsection{BNPs show a safe toxicological profile}

Side effects induced by HCQ and CLB have been well described in the literature $[37,38]$ and were also evident in our experiments on healthy mice.

The reduction of side-effects was addressed by including $\mathrm{CLB}+\mathrm{HCQ}$ drugs in BNPs produced from biocompatible and biodegradable polymers. The development of these nanoparticles with an average diameter of $250 \mathrm{~nm}$ as drug-delivery agents has several advantages, including specific targeting via receptormediated mechanisms and effective penetration of the tumor microenvironment [19]. BNP2 in particular can transport and release into tumor B-cells enough amount of drugs to kill cancer cells, thus overcoming multidrug resistance found in several B-cell disorders [39].

The toxic effects of the intra-peritoneal injection of BNPs were evaluated in groups of five C57/BL mice receiving 8 injections of saline, 8 injections of BNP2 (containing $400 \mu \mathrm{g}$ of CLB and HCQ), or 4 injections of free HCQ+CLB (400 $\mu \mathrm{g}$ each). We have previously shown that 8 injections of free drugs killed all the animals [18].

The mice were followed for 28 days. Animal survival, total body weight, circulating cells, and several tissue markers in the blood were analyzed. All the animals survived during the experiment but the free drugstreated mice showed a strong reduction in their body weight, with a median of about $20 \%$. Blood samples were collected 3 days after the end of the treatments in order to evaluate complete blood count, hemoglobin, urea, aspartate transaminase (AST), alanine transaminase (ALT), alkaline phosphatase (ALP), lactatedehydrogenase $(\mathrm{LDH})$, creatine phosphokinase (CPK), creatinine, and aldolase concentrations (Table 2). We did not find any significant differences during any of the experiments between the controls and animals receiving 8 injections of BNP2; only platelets seemed to have increased after the treatments, but remained in a physiological range. On the contrary, the animals receiving only 4 injections of free $\mathrm{HCQ}+\mathrm{CLB}$ showed
Table 2 Toxicological studies

\begin{tabular}{cccc}
\hline & Saline & BNP2 & HCQ/CLB \\
\hline $\begin{array}{c}\text { Body weight } \\
(\% \text { variation })\end{array}$ & -2.31 & -3.29 & $-20.61^{* \S}$ \\
WBC $\left(10^{3} / \mathrm{mm}^{3}\right)$ & $3.15 \pm 1.08$ & $2.13 \pm 0.4$ & $0.58 \pm 0.33^{* \S}$ \\
LYMPHOCYTES & $2.65 \pm 1.03$ & $1.60 \pm 0.17$ & $0.30 \pm 0.18^{* \S}$ \\
$\left(10^{3} / \mathrm{mm}^{3}\right)$ & & & \\
MONOCYTES & $0.18 \pm 0.05$ & $0.20 \pm 0.17$ & $0.08 \pm 0.18$ \\
$\left(10^{3} / \mathrm{mm}^{3}\right)$ & & & \\
GRANULOCYTES & $0.33 \pm 0.05$ & $0.33 \pm 0.06$ & $0.20 \pm 0.1$ \\
$\left(10^{3} / \mathrm{mm}^{3}\right)$ & $7.42 \pm 1$ & $5.52 \pm 1.47$ & $4.87 \pm 0.52^{*}$ \\
RBC $\left(10^{6} / \mathrm{mm}^{3}\right)$ & $688.00 \pm$ & $464.5 \pm 93.3$ \\
PLT $\left(10^{3} / \mathrm{mm}^{3}\right)$ & $532.25 \pm 73.4$ & $27.22^{* \S}$ & \\
Hemoglobin $(\mathrm{g} / \mathrm{L})$ & $11 \pm 0.6$ & $11 \pm 0.4$ & $9.0 \pm 1.3^{* \S}$ \\
Urea $(\mathrm{mg} / \mathrm{dL})$ & $29 \pm 5$ & $28 \pm 9$ & $28 \pm 7$ \\
Creatinine $(\mathrm{mg} / \mathrm{dL})$ & $0.24 \pm 0.06$ & $0.20 \pm 0.18$ & $0.14 \pm 0.02^{*}$ \\
AST $(\mathrm{IU} / \mathrm{L})$ & $152 \pm 52$ & $146 \pm 16$ & $182 \pm 35$ \\
ALT $(\mathrm{IU} / \mathrm{L})$ & $26.8 \pm 6.0$ & $17.0 \pm 5.3$ & $20.3 \pm 9.6$ \\
ALP $(\mathrm{IU} / \mathrm{L})$ & $34.0 \pm 10.2$ & $26.7 \pm 10.0$ & $18.5 \pm 5.8^{*}$ \\
LDH $(\mathrm{IU} / \mathrm{L})$ & $645 \pm 82.8$ & $435 \pm 98.6$ & $495 \pm 41.5^{*}$ \\
CPK $(\mathrm{IU} / \mathrm{L})$ & $2,084 \pm 413$ & $1,143 \pm 564$ & $1,587 \pm 223$ \\
Aldolase $(\mathrm{IU} / \mathrm{L})$ & $9 \pm 4$ & $11 \pm 2$ & $16 \pm 5^{*} \S$ \\
\hline
\end{tabular}

Total body weight, red blood cells (RBC), white blood cells (WBC), platelets (PLT), and other plasma parameters from treated and untreated mice were compared. ${ }^{*} p<0.05$ vs. control; ${ }^{\S} p<0.05$ vs. BNP2.

a reduction in white blood cells, mainly due to the low number of circulating lymphocytes, and a significant reduction in erythrocytes.

Simultaneously, we observed reduced concentration of hemoglobin, creatinine, ALP, and LDH, with increased values of aldolase (Table 2).

\subsection{Development of a disseminated leukemia model using MEC1 cells}

A xenograft model of human CLL was previously described by Bertilaccio et al. [40], who challenged $\mathrm{Rag}^{-/-} \gamma_{\mathrm{c}}^{-/-}$mice intravenously or subcutaneously with $10^{7} \mathrm{MEC} 1$ cells. Unfortunately, we were unable to repeat these results in SCID mice; in fact, the intravenous injection of $10^{7}$ cells rapidly killed all the animals by causing respiratory problems. On the other hand, 
subcutaneous cell injection induced only the formation of a localized tumor mass at the site of injection without colonizing other tissues and causing the death of the animals in about 70 days (Fig. 3).

However, an intravenous injection of only $5 \times 10^{5}$ MEC1 cells $24 \mathrm{~h}$ after a pre-treatment with cyclophosphamide $(200 \mathrm{mg} / \mathrm{kg}$ intraperitoneally), which is known to reduce immune effects, in particular via NK cells [41,42], resulted in a diffuse leukemia model characterized by the colonization of different organs and the blood. In details, the biodistribution pattern of the MEC1 cells was evaluated by immunohistochemical analysis by staining the tissues with $\mathrm{H} \& \mathrm{E}$ and detecting human B cells with an anti-human CD45 antibody 28 days after MEC1 injection (Fig. 4(a)). MEC1 cells were detected in the liver, spleen, kidney, bone marrow, spinal cord, and brain. Moreover, the accumulation of MEC1 cells in the bloodstream of the mice was confirmed by cytometric analysis using anti-human CD19 and anti-human CD45 antibodies. The presence of human B-cells was detected from the $20^{\text {th }}$ day after tumor cell injection (Fig. 4(b)).

All animals died between 30 and 37 days after tumor cell injection (Fig. 3), which confirmed the development of a very reproducible and very aggressive leukemia human/SCID model, which was useful for the characterization of the therapeutic effect of the targeted nanoparticles and also for the development of new recombinant antibodies, as already shown for other B-cell malignancies [24, 30, 43].

\subsection{Therapeutic effect of BNP2 in a disseminated leukemia human/mouse model}

BNP2 has already shown its ability to target cancer B-cells in vivo as well as a potential efficacy in the treatment of tumor-bearing mice, as already evidenced in other B-cell xenografts $[18,19]$. To study the efficacy of BNP2 in the treatment of the human/SCID leukemia model, MEC1 cells were injected in SCID mice, divided into 8 groups of $6-8$ animals per group, and followed up for 120 days (Fig. 5).

Group 1 did not receive any treatment; all mice died within 30-40 days after tumor cell injection with a median survival time of 33.5 days. The therapeutic protocol followed our previous data and derived from the toxicological profile obtained with free HCQ+CLB.

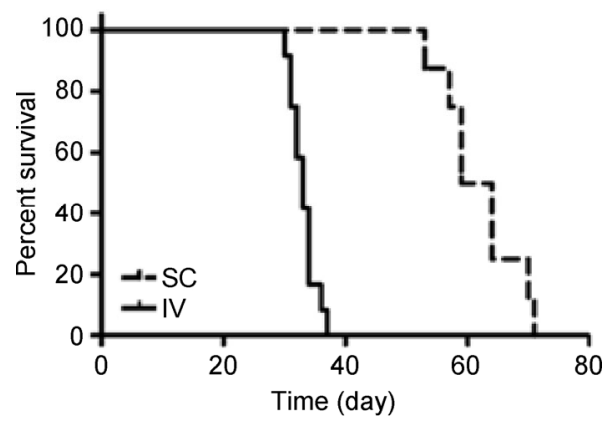

Figure 3 Development of human/SCID leukemia model. MEC1 were injected subcutaneously (SC, $10^{7}$ cells) or intravenously (IV, $5 \times 10^{5}$ cells) after cyclophosphamide pre-treatment. Animal survival was studied and reported as Kaplan Mayer curves.

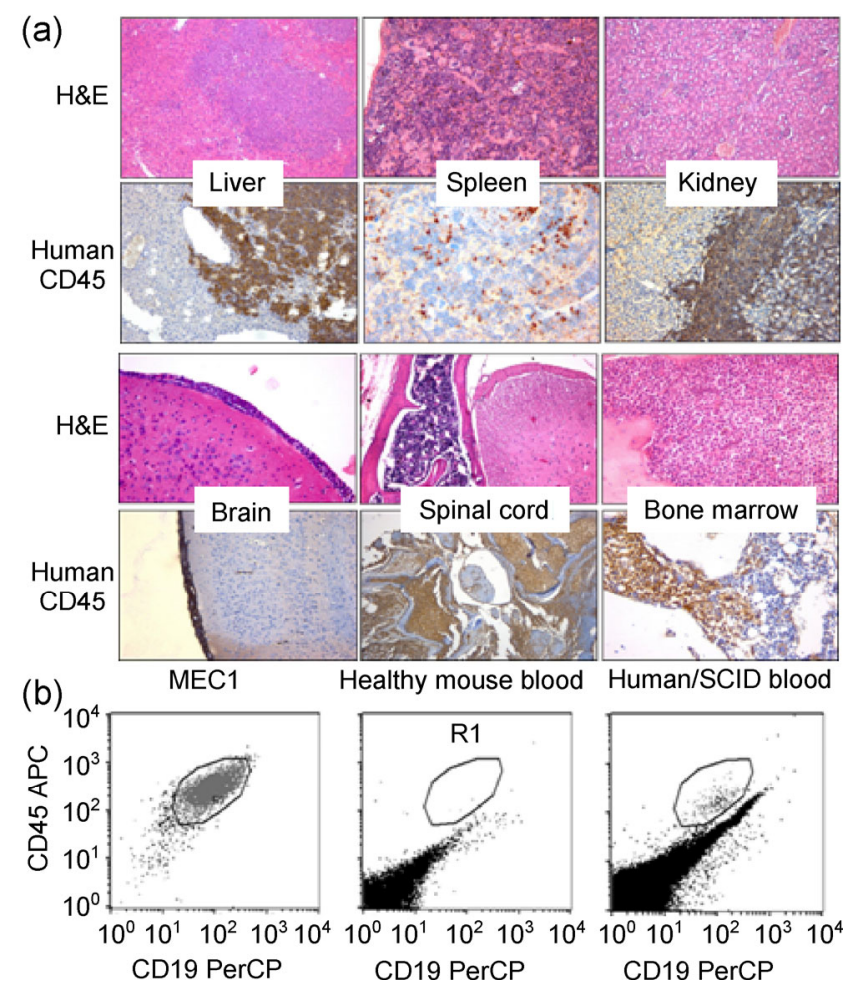

Figure 4 Characterization of diffuse leukemia model in SCID mice. MEC1 cells $\left(5 \times 10^{5}\right)$ were injected intravenously in SCID mice and the distribution of human tumor cells was analyzed after 28 days by H\&E and by exploiting human CD45 (a). Original magnification $200 \times$. (b) Human tumor B-cells were also detected in circulation by FACS analysis using labeled anti-CD45 and anti-CD19 mAbs.

Thus, group 2 and group 3 both received $80 \mu \mathrm{L}$ of BNP2 (corresponding to $400 \mu \mathrm{g}$ of each encapsulated chemotherapeutic agent targeted via anti-CD20 antibody) for 8 times in 17 days from the 1st and the 4th day after MEC1 cell injection, respectively. The overall survival of group 2 was 83 days and 3 out of 


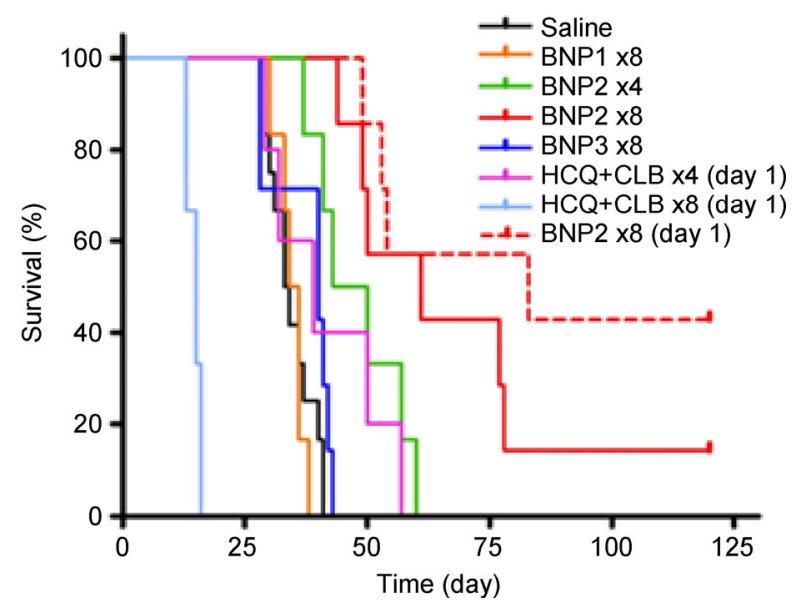

Figure 5 Therapeutic effect of BNPs and HCQ+CLB. SCID mice $\left(n=5-10\right.$ per group) received $\left(5 \times 10^{6}\right) \mathrm{MEC} 1$ cells intravenously and $\mathrm{BNP} 1, \mathrm{BNP} 2, \mathrm{BNP} 3$, or HCQ $+\mathrm{CLB}$ as described in the results; animal survival was represented as a Kaplan Mayer curve.

7 mice were cured at the end of the study $(\mathrm{BNP} 2 \times 8$ (day 1) vs. untreated: $p<0.0001 ; \mathrm{BNP} 2 \times 8$ (day 1$)$ vs. $\mathrm{BNP} 1 \times 8: p<0.0002 ; \mathrm{BNP} 2 \times 8($ day 1$)$ vs. $\mathrm{BNP3} \times 8: p$ $<0.0001$; BNP2 $\times 8$ (day 1) vs. HCQ+CLB: $p<0.01$; $\mathrm{BNP} 2 \times 8($ day 1$)$ vs. $\mathrm{BNP} 2 \times 4: p<0.03 ; \mathrm{BNP} 2 \times 8($ day 1$)$ vs. BNP2 $\times 8$ (day 4$)$ : not significant). Group 3 received the same treatment but starting from day 4 , resulting in an overall survival of 61 days and 1 out of 7 mice was cured (BNP2 $\times 8$ (day 4) vs. untreated: $p<0.0001$; $\mathrm{BNP} 2 \times 8($ day 4$)$ vs. $\mathrm{BNP1} \times 8: p<0.0002 ; \mathrm{BNP} 2 \times 8$ (day 4 ) vs. BNP3 $\times 8$ : $p<0.0001$; BNP2 $\times 8$ (day 4 ) vs. HCQ+CLB: $p<0.03$; BNP2 $\times 8$ (day 4 ) vs. BNP2 $\times 4$ : $p<0.04)$. These results reveal the ability of BNP2 to treat this aggressive human/mouse leukemia model with a better outcome when the treatment was started at the early stage of the pathology.

Group 4 received only 4 injections of $80 \mu \mathrm{L}$ of BNP2 in 8 days starting from the 4th day after cell injection. This treatment improved the overall survival by about 13.5 days (BNP2 $\times 4$ vs. Untreated: $p<0.002$; BNP2 $\times$ 4 vs. HCQ+CLB: $p<0.03)$.

Group 5 and group 6 received 8 injections of $80 \mu \mathrm{L}$ of BNP1 and BNP3, respectively. Both these treatments did not significantly increase the survival of the mice, thus demonstrating both the safety of the BNPs and the inability of BNP3 to bind to cancer cells due to the absence of the anti-CD20 antibody on the surface of these particles, as previously demonstrated by our group [19]. In vitro, untargeted nanoparticles
(BNP3) showed cytotoxicity, but their effect was not confirmed in vivo. This was probably due to the blood flow (for circulating tumor cells) and reduced residence time of untargeted nanoparticles in the tumor microenvironment.

Finally, group 7 received 8 injections of HCQ+CLB (400 $\mu \mathrm{g}$ each) in 17 days starting from day 1 . This treatment improved the survival by 2 days and showed that BNP2 (groups 2) was more effective than free drugs in the treatment of this aggressive human/ mouse leukemia model.

In group 8, 3 animals received 8 injections of free drugs but all the mice died due to toxicity in less than 20 days, as already described [18].

\section{Conclusions}

In conclusion, the results of the present study revealed that anti-CD20 nanoparticles containing HCQ+CLB can be effective as a single therapeutic agent in controlling aggressive leukemia in the new disseminated model. We also provide a rationale for adopting this therapeutic approach for the treatment of other B-cell disorders with BNP2 or different types of tumors by using other monoclonal antibodies to specifically deliver cytotoxic agent-loaded nanoparticles in cancer cells.

\section{Acknowledgements}

This study has been made possible by research grants from Italian Association for Cancer Research (AIRC Project No. 12965/2012), Italian Ministry of Health (Nos. GR-2011-02346826 and GR-2011- 02347441), Fondazione Casali - Trieste, Italy and Stiftung Foundation - Liechtenstein. Nanoparticles fabrication at LNK Chemsolutions, USA, was possible in part by Grant 2R44CA135906-02 (SBIR Phase II) from the National Institutes of Health (USA) to Ruben Spretz, Gustavo Larsen, Sandra Noriega and Luis Núñez.

Conflict-of-interest disclosure: Ruben Spretz, Gustavo Larsen, Sandra Noriega and Luis Núñez working in Biotarget Inc. and LNK Chemsolutions LLC have commercial interests in the particle systems described in this work. No conflicts of interest for the other authors. 
Electronic Supplementary Material: Supplementary material (CLL patients' characterization (confocal microscopy, cytometry, sequencing, killing test)) is available in the online version of this article at http:// dx.doi.org/10.1007/s12274-015-0935-3.

\section{References}

[1] Hallek, M.; Cheson, B. D.; Catovsky, D.; Caligaris-Cappio, F.; Dighiero, G.; Döhner, H.; Hillmen, P.; Keating, M. J.; Montserrat, E.; Rai, K. R. et al. Guidelines for the diagnosis and treatment of chronic lymphocytic leukemia: A report from the International Workshop on Chronic Lymphocytic Leukemia updating the National Cancer Institute-Working Group 1996 guidelines. Blood 2008, 111, 5446-5456.

[2] Zenz, T.; Eichhorst, B.; Busch, R.; Denzel, T.; Häbe, S.; Winkler, D.; Bühler, A.; Edelmann, J.; Bergmann, M.; Hopfinger, G. et al. TP53 mutation and survival in chronic lymphocytic leukemia. J. Clin. Oncol. 2010, 28, 4473-4479.

[3] Puente, X. S.; Pinyol, M.; Quesada, V.; Conde, L.; Ordóñez, G. R.; Villamor, N.; Escaramis, G.; Jares, P.; Beà, S.; González-Díaz, M. et al. Whole-genome sequencing identifies recurrent mutations in chronic lymphocytic leukaemia. Nature 2011, 475, 101-105.

[4] Fabbri, G.; Rasi, S.; Rossi, D.; Trifonov, V.; Khiabanian, H.; Ma, J.; Grunn, A.; Fangazio, M.; Capello, D.; Monti, S. et al. Analysis of the chronic lymphocytic leukemia coding genome: Role of NOTCH1 mutational activation. J. Exp. Med. 2011, 208, 1389-1401.

[5] Rossi, D.; Rasi, S.; Spina, V.; Bruscaggin, A.; Monti, S.; Ciardullo, C.; Deambrogi, C.; Khiabanian, H.; Serra, R.; Bertoni, F. et al. Integrated mutational and cytogenetic analysis identifies new prognostic subgroups in chronic lymphocytic leukemia. Blood 2013, 121, 1403-1412.

[6] Rossi, D.; Rasi, S.; Fabbri, G.; Spina, V.; Fangazio, M.; Forconi, F.; Marasca, R.; Laurenti, L.; Bruscaggin, A.; Cerri, M. et al. Mutations of NOTCH1 are an independent predictor of survival in chronic lymphocytic leukemia. Blood 2012, 119, 521-529.

[7] Wang, L. L.; Lawrence, M. S.; Wan, Y. Z.; Stojanov, P.; Sougnez, C.; Stevenson, K.; Werner, L.; Sivachenko, A.; DeLuca, D. S.; Zhang, L. et al. SF3B1 and other novel cancer genes in chronic lymphocytic leukemia. N. Engl. J. Med. 2011, 365, 2497-2506.

[8] Dreger, P.; Schetelig, J.; Andersen, N.; Corradini, P.; van Gelder, M.; Gribben, J.; Kimby, E.; Michallet, M.; Moreno, C.; Stilgenbauer, S. et al. Managing high-risk CLL during transition to a new treatment era: Stem cell transplantation or novel agents? Blood 2014, 124, 3841-3849.
[9] Siddiqi, T.; Rosen, S. T. Novel biologic agents for nonhodgkin lymphoma and chronic lymphocytic leukemia-Part 1. Oncology (Williston Park) 2015, 29, 198-203.

[10] Woyach, J. A.; Johnson, A. J. Targeted therapies in CLL: Mechanisms of resistance and strategies for management. Blood 2015, 126, 471-477.

[11] Jain, N.; O'Brien, S. Initial treatment of CLL: Integrating biology and functional status. Blood 2015, 126, 463-470.

[12] Hallek, M. Chronic lymphocytic leukemia: 2013 update on diagnosis, risk stratification and treatment. Am. J. Hematol. 2013, 88, 803-816.

[13] Dawidczyk, C. M.; Kim, C.; Park, J. H.; Russell, L. M.; Lee, K. H.; Pomper, M. G.; Searson, P. C. State-of-the-art in design rules for drug delivery platforms: Lessons learned from FDA-approved nanomedicines. J. Control. Release 2014, 187, 133-144.

[14] Bartlett, D. W.; Su, H. L.; Hildebrandt, I. J.; Weber, W. A.; Davis, M. E. Impact of tumor-specific targeting on the biodistribution and efficacy of siRNA nanoparticles measured by multimodality in vivo imaging. Proc. Natl. Acad. Sci. USA 2007, 104, 15549-15554.

[15] Sanna, V.; Pala, N.; Sechi, M. Targeted therapy using nanotechnology: Focus on cancer. Int. J. Nanomedicine 2014, 9, 467-483.

[16] Chattopadhyay, N.; Fonge, H.; Cai, Z. L.; Scollard, D.; Lechtman, E.; Done, S. J.; Pignol, J. P.; Reilly, R. M. Role of antibody-mediated tumor targeting and route of administration in nanoparticle tumor accumulation in vivo. Mol. Pharmaceutics 2012, 9, 2168-2179.

[17] Wang, A. Z.; Langer, R.; Farokhzad, O. C. Nanoparticle delivery of cancer drugs. Annu. Rev. Med. 2012, 63, 185-198.

[18] Mezzaroba, N.; Zorzet, S.; Secco, E.; Biffi, S.; Tripodo, C.; Calvaruso, M.; Mendoza-Maldonado, R.; Capolla, S.; Granzotto, M.; Spretz, R. et al. New potential therapeutic approach for the treatment of B-cell malignancies using chlorambucil/hydroxychloroquine-loaded anti-CD20 nanoparticles. PLoS One 2013, 8, e74216.

[19] Capolla, S.; Garrovo, C.; Zorzet, S.; Lorenzon, A.; Rampazzo, E.; Spretz, R.; Pozzato, G.; Núñez, L.; Tripodo, C.; Macor, P. et al. Targeted tumor imaging of anti-CD20polymeric nanoparticles developed for the diagnosis of B-cell malignancies. Int. J. Nanomedicine 2015, 10, 4099-4109.

[20] Stacchini, A.; Aragno, M.; Vallario, A.; Alfarano, A.; Circosta, P.; Gottardi, D.; Faldella, A.; Rege-Cambrin, G.; Thunberg, U.; Nilsson, K. et al. MEC1 and MEC2: Two new cell lines derived from B-chronic lymphocytic leukaemia in prolymphocytoid transformation. Leuk. Res. 1999, 23, 127-136.

[21] Dereani, S.; Macor, P.; D’Agaro, T.; Mezzaroba, N.; Dal-Bo, M.; Capolla, S.; Zucchetto, A.; Tissino, E.; Del Poeta, G.; Zorzet, S. et al. Potential therapeutic role of antagomiR17 
for the treatment of chronic lymphocytic leukemia. $J$. Hematol. Oncol. 2014, 7, 79.

[22] Biffi, S.; Garrovo, C.; Macor, P.; Tripodo, C.; Zorzet, S.; Secco, E.; Tedesco, F.; Lorusso, V. In vivo biodistribution and lifetime analysis of Cy5. 5-Conjugated rituximab in mice bearing lymphoid tumor xenograft using time-domain near-infrared optical imaging. Mol. Imaging 2008, 7, 272-282.

[23] Marín, G. H.; Mansilla, E.; Mezzaroba, N.; Zorzet, S.; Núñez, L.; Larsen, G.; Tau, J. M.; Maceira, A.; Spretz, R.; Mertz, C. et al. Exploratory study on the effects of biodegradable nanoparticles with drugs on malignant $\mathrm{B}$ cells and on a human/mouse model of Burkitt lymphoma. Curr. Clin. Pharmacol. 2010, 5, 246-250.

[24] Macor, P.; Secco, E.; Mezzaroba, N.; Zorzet, S.; Durigutto, P.; Gaiotto, T.; De Maso, L.; Biffi, S.; Garrovo, C.; Capolla, $\mathrm{S}$. et al. Bispecific antibodies targeting tumor-associated antigens and neutralizing complement regulators increase the efficacy of antibody-based immunotherapy in mice. Leukemia 2015, 29, 406-414.

[25] Zabucchi, G.; Soranzo, M. R.; Menegazzi, R.; Vecchio, M.; Knowles, A.; Piccinini, C.; Spessotto, P.; Patriarca, P. Eosinophil peroxidase deficiency: Morphological and immunocytochemical studies of the eosinophil-specific granules. Blood 1992, 80, 2903-2910.

[26] Tripodo, C.; Florena, A. M.; Macor, P.; Di Bernardo, A.; Porcasi, R.; Guarnotta, C.; Ingrao, S.; Zerilli, M.; Secco, E.; Todaro, M. et al. P-selectin glycoprotein ligand-1 as a potential target for humoral immunotherapy of multiple myeloma (Supplementry material). Curr. Cancer Drug Tar. 2009, 9, 617-625.

[27] Mendoza-Maldonado, R.; Paolinelli, R.; Galbiati, L.; Giadrossi, S.; Giacca, M. Interaction of the retinoblastoma protein with orc1 and its recruitment to human origins of DNA replication. PLoS One 2010, 5, e13720.

[28] Macor, P.; Tripodo, C.; Zorzet, S.; Piovan, E.; Bossi, F.; Marzari, R.; Amadori, A.; Tedesco, F. In vivo targeting of human neutralizing antibodies against CD55 and CD59 to lymphoma cells increases the antitumor activity of rituximab. Cancer Res. 2007, 67, 10556-10563.

[29] Florena, A. M.; Tripodo, C.; Iannitto, E.; Porcasi, R.; Ingrao, S.; Franco, V. Value of bone marrow biopsy in the diagnosis of essential thrombocythemia. Haematologica 2004, 89, 911-919.

[30] Bertilaccio, M. T. S.; Scielzo, C.; Simonetti, G.; Ten Hacken, E.; Apollonio, B.; Ghia, P.; Caligaris-Cappio, F. Xenograft models of chronic lymphocytic leukemia: Problems, pitfalls and future directions. Leukemia 2013, 27, 534-540.

[31] Kalil, N.; Cheson, B. D. Management of chronic lymphocytic leukaemia. Drugs Aging 2000, 16, 9-27.
[32] Zhou, Y.; Hileman, E. O.; Plunkett, W.; Keating, M. J.; Huang, P. Free radical stress in chronic lymphocytic leukemia cells and its role in cellular sensitivity to ROS-generating anticancer agents. Blood 2003, 101, 4098-4104.

[33] Amaravadi, R. K.; Lippincott-Schwartz, J.; Yin, X.-M.; Weiss, W. A.; Takebe, N.; Timmer, W.; DiPaola, R. S.; Lotze, M. T.; White, E. Principles and current strategies for targeting autophagy for cancer treatment. Clin. Cancer Res. 2011, 17, 654-666.

[34] Lagneaux, L.; Delforge, A.; Dejeneffe, M.; Massy, M.; Bernier, M.; Bron, D. Hydroxychloroquine-induced apoptosis of chronic lymphocytic leukemia involves activation of caspase- 3 and modulation of Bcl-2/bax/ratio. Leuk Lymphoma 2002, 43, 1087-1095.

[35] Mansilla, E.; Marin, G. H.; Nuñez, L.; Drago, H.; Sturla, F.; Mertz, C.; Rivera, L.; Ichim, T.; Riordan, N.; Raimondi, C. The lysosomotropic agent, hydroxychloroquine, delivered in a biodegradable nanoparticle system, overcomes drug resistance of B-chronic lymphocytic leukemia cells in vitro. Cancer Biother. Radiopharm. 2010, 25, 97-103.

[36] Rahim, R.; Strobl, J. S. Hydroxychloroquine, chloroquine, and all-trans retinoic acid regulate growth, survival, and histone acetylation in breast cancer cells. Anti-Cancer Drugs 2009, 20, 736-745.

[37] Tehrani, R.; Ostrowski, R. A.; Hariman, R.; Jay, W. M. Ocular toxicity of hydroxychloroquine. Semin. Ophthalmol. 2008, 23, 201-209.

[38] Stein, M.; Bell, M. J.; Ang, L. C. Hydroxychloroquine neuromyotoxicity. J. Rheumatol. 2000, 27, 2927-2931.

[39] Rao, D. A.; Forrest, M. L.; Alani, A. W.; Kwon, G. S.; Robinson, J. R. Biodegradable PLGA based nanoparticles for sustained regional lymphatic drug delivery. J. Pharm. Sci. 2010, 99, 2018-2031.

[40] Bertilaccio, M. T. S.; Scielzo, C.; Simonetti, G.; Ponzoni, M.; Apollonio, B.; Fazi, C.; Scarfò, L.; Rocchi, M.; Muzio, M.; Caligaris-Cappio, F. et al. A novel $\operatorname{Rag} 2^{-/-} \gamma_{\mathrm{c}}^{-/-}$-xenograft model of human CLL. Blood 2010, 115, 1605-1609.

[41] Nonaka, Y.; Ishibashi, H.; Nakai, M.; Shibata, H.; Kiso, Y.; Abe, S. Effects of the antlered form of Ganoderma lucidum on tumor growth and metastasis in cyclophosphamide-treated mice. Biosci. Biotechnol. Biochem. 2008, 72, 1399-1408.

[42] Zorzet, S.; Perissin, L.; Rapozzi, V.; Giraldi, T. Restraint stress reduces the antitumor efficacy of ciclophosphamide in tumor-bearing mice. Brain Behav. Immun. 1998, 12, 23-33.

[43] Macor, P.; Secco, E.; Zorzet, S.; Tripodo, C.; Celeghini, C.; Tedesco, F. An update on the xenograft and mouse models suitable for investigating new therapeutic compounds for the treatment of B-cell malignancies. Curr. Pharm. Des. 2008, 14, 2023-2039. 